\title{
Treatment-related adverse effects with TKIs in patients with advanced or radioiodine refractory differentiated thyroid carcinoma: a systematic review and meta-analysis
}

This article was published in the following Dove Medical Press journal: Cancer Management and Research

\author{
Shi-Tong Yu ${ }^{1, *}$ \\ Jun-Na Ge $\mathrm{e}^{1, *}$ \\ Jing-Yi Luo ${ }^{2, *}$ \\ Zhi-Gang Wei' \\ Bai-Hui Sun' \\ Shang-Tong Lei' \\ 'Department of General Surgery, \\ Nanfang Hospital, Southern Medical \\ University, Guangzhou, Guangdong, \\ China; ${ }^{2}$ State Key Laboratory \\ of Ophthalmology, Zhongshan \\ Ophthalmic Center, Sun Yat-sen \\ University, Guangzhou, Guangdong, \\ China \\ *These authors contributed equally to \\ this work
}

Background: Tyrosine kinase inhibitors (TKIs) have been administered to advanced or radioiodine refractory differentiated thyroid carcinoma (RR-DTC) patients for years. We performed a pooled analysis to explore the frequency of severe adverse effects in advanced or RR-DTC patients treated with sorafenib and lenvatinib.

Methods: We performed a comprehensive search of computerized databases, including PubMed, Web of Science, Ovid, EMASE, and the Cochrane Library, from the drugs' inception to July 2018 to identify clinical trials. All grade and severe adverse events (AEs; grade $\geq 3$ ) were analyzed. This meta-analysis was conducted in accordance with PRISMA guidelines.

Results: In total, seve studies published from 2012-2018 with 657 patients were eligible for this study. We included two studies (238 patients) that received $200 \mathrm{mg}$ sorafenib twice and five studies (419 patients) that received $24 \mathrm{mg}$ lenvatinib daily. The frequency of AEs was different among the two drugs. Patients in the sorafenib group had a significantly higher frequency of all grade hand-foot syndrome, hypocalcemia, rash, elevated alanine aminotransferase (ALT), and elevated aspartate aminotransferase (AST). Conversely, the lenvatinib group experienced more frequent all grade voice change, hypertension, nausea, and vomiting compared with those with sorafenib. For grade $\geq 3$ adverse effects, hand-foot syndrome, hypocalcemia, and elevated ALT were more frequent in sorafenib-treated patients. Moreover, lenvatinib-treated patients had a significantly higher incidence of severe weight loss, hypertension, and nausea.

Conclusion: Significant differences in common adverse effects, such as all-grade and severe AEs, were detected between sorafenib and lenvatinib in the current study. Early intervention and management of treatment-related AEs (TRAEs) can minimize the impact on patients' quality-of-life, and avoid unnecessary dose reductions and treatment-related discontinuations. Keywords: sorafenib, lenvatinib, radioiodine-refractory differentiated thyroid carcinoma, RRDTC, tyrosine kinase inhibitors, TKIs, adverse effects

\section{Introduction}

Thyroid cancer is one of the most frequent malignancies of the endocrine system, with an increasing trend in recent decades. ${ }^{1}$ Differentiated thyroid cancer (DTC) comprises over $95 \%$ of all thyroid cancers, whereas the rest are medullary and anaplastic thyroid cancer.

Classical treatments for DTC include surgery, radioactive iodine (RAI) therapy of remnant thyroid ablation, and thyroid-stimulating hormone (TSH) suppression therapy. ${ }^{2}$ Most DTC patients who receive these treatments have a relatively good prognosis,
Correspondence: Shang-Tong Lei Department of General Surgery, Nanfang Hospital, Southern Medical University, No. 1838 North Guangzhou Avenue, Guangzhou, Guangdong, China

Tel +862061641888

Fax +862061641866

Email leisht781920@I63.com 
with $>95 \%$ 5-year overall survival (OS) rates. For patients with distant metastasis, the 5-year OS decreases to $50 \%$. However, for patients with inadequate tumor responses to RAI, the 5-year OS drops to $19 \%{ }^{2}$

Recently, small-molecule tyrosine kinase inhibitors (TKIs) have become new treatment options for advanced or radioiodine refractory differentiated thyroid cancer (RRDTC) based on several clinical trials. TKIs were designed to target multiple sites of the kinase cascade, which promotes cell growth, expansion, and metastasis. ${ }^{3}$ Several TKIs, including sorafenib, lenvatinib, vandetanib, and cabozantinib, have been investigated and showed clinical values in patients with advanced or metastatic DTC. ${ }^{4-8}$ For sorafenib and lenvatinib, the FDA approved the treatment for advanced or RR-DTC in November 2013 and February 2015, respectively.

However, the utility of VEGFR-TKIs was restrained by their side effects, and the underlying mechanism is still unknown. Furthermore, the difference in toxicity between sorafenib and lenvatinib has not been fully elucidated. Therefore, it is important and necessary to select optimal TKIs with acceptable toxicological properties, lowering the influence on patients' quality-of-life (QoL). Thus, in the current study, we conducted a pooled analysis of adverse events (AEs) based on data extracted from clinical studies of patients with advanced or RR-DTC.

\section{Materials and methods}

\section{Study identification}

This meta-analysis was conducted in accordance with PRISMA guidelines. A comprehensive search of computerized databases to include relevant studies published in English between January 2008 and May 2018 was performed, including PubMed, Web of Science, Ovid, EMASE, and the Cochrane Library, encompassing the period from the drugs' inspection on July 2018. The search keywords were "sorafenib", "lenvatinib", and "differentiated thyroid cancer" (Supplementary materials). Abstracts from the American Society of Clinical Oncology (ASCO) meeting were handsearched for updated data and new studies. After these papers were screened and reviewed, duplicated data and irrelevant papers were not included in the study. Reference lists were also hand-searched to identify new articles.

The study inclusion criteria were as follows: 1) patients $\geq 18$ years with advanced or RR-DTC; 2) interventions: sorafenib or lenvatinib, with FDA-approved doses, not combined with other therapies; 3) sufficient data reported on treatment-related AEs (TRAEs), including all information regarding all-grade and grade $\geq 3$; and 4) written in English.
All case reports, letters, commentaries, and reviews were excluded from the study.

\section{Data extraction and quality control}

Two reviewers (S-T Yu and J-N Ge) independently assessed the methodological quality of each study using the risk of bias method recommended by the Cochrane Collaboration. Risk of bias that may affect the cumulative evidence was also assessed by these two reviewers. Differences were solved by discussion or through consulting with the senior investigator (S-T Lei).

The first author's name, publication year, TKI drug (sorafenib or lenvatinib), number of patients for all-grade and grade $\geq 3$ toxicity (hand-foot syndrome, nausea, diarrhea, fatigue, vomiting, hypertension, hypocalcemia, rash, elevated alanine aminotransferase (ALT), elevated aspartate aminotransferase (AST), voice change, mucositis, anorexia, number of patients experiencing treatment-related death (TRD), and withdrawal resulting from severe toxicity) were evaluated. Safety data were retrieved from patients receiving $200 \mathrm{mg}$ sorafenib twice or $24 \mathrm{mg}$ lenvatinib daily according to the FDA-recommended dose. Studies were independently evaluated by two reviewers for the aforementioned inclusion criteria.

\section{Statistical analysis}

All statistical analysis was performed on GraphPad Prism (version 7.0; GraphPad Software, San Diego, CA, USA). Fisher's exact or chi-square tests were used to compare the frequencies of AEs among DTC patients. All tests were twotailed, and statistical significance was considered at $P<0.05$.

\section{Results \\ Characteristics of the original selected studies}

Based on our inclusion criteria, we identified seven studies regarding TKIs treating advanced or RR-DTC in the current study (Figure 1). From seven studies conducted between 2012 and 2018, 657 patients were included in the current study. The sample size ranged from 12 to 261 . The patients in two studies (238 patients) received sorafenib, ${ }^{4,9}$ and patients in five studies (419 patients) received lenvatinib. ${ }^{8,10-13}$ The basic characteristics of the included studies are shown in Table S1.

\section{Study quality assessment and risk bias}

No major flaws were identified in the assessment of bias risk in the included studies (Table S2; Figure S1). However, the absence of blinded interventions was a common caveat. 


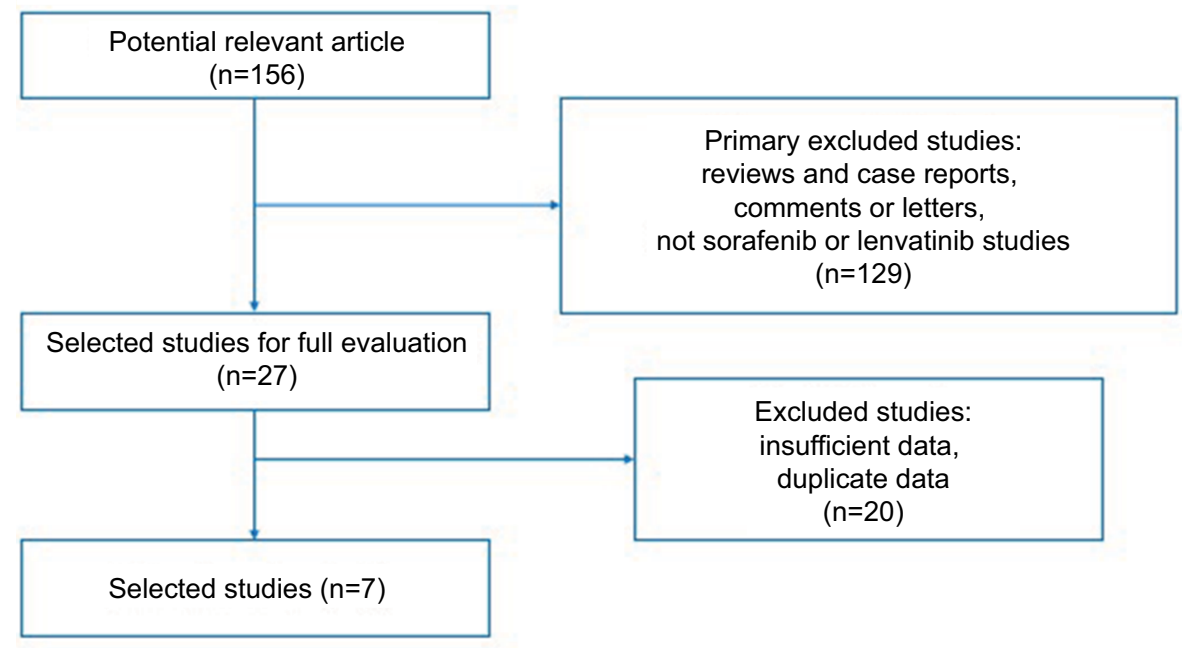

Figure I Flow chart of the selection of studies included in this pooled study.

\section{Frequency of all-grade TRAEs between sorafenib and lenvatinib}

We analyzed the incidence and odds ratios (ORs) of TRAEs by TKI in patients with DTC. The incidence of all grade handfoot syndrome was higher in those with sorafenib (75.6\%) than lenvatinib (32.1\%). The difference between incidence was significantly higher in sorafenib vs lenvatinib $(\mathrm{OR}=6.56$, $95 \%$ CI=4.53-9.48, $P<0.0001$; Figure 2A).

Similarly, the incidence of all grade hypocalcemia was higher in patients treated with sorafenib (22.7\%) compared to those with lenvatinib (6.9\%). The difference in incidence was significant in sorafenib vs lenvatinib (OR=3.96, 95\% $\mathrm{CI}=2.25-6.98, P<0.0001$; Figure 2B).

Meanwhile, the patients treated with sorafenib (50.8\%) had a higher incidence of all grade rash compared with those with lenvatinib (11.6\%), and the difference remained statistically significant $(\mathrm{OR}=5.39,95 \% \mathrm{CI}=3.56-8.18, P<0.0001$; Figure 2C).

The frequency of all grade voice change with sorafenib was $12.1 \%$ and it was $46.1 \%$ in lenvatinib. The difference in frequency was statistically significant in sorafenib vs lenvatinib (OR=0.49, 95\% $\mathrm{CI}=0.30-0.79, P=0.003$; Figure 2D). Likewise, patients treated with sorafenib had a lower frequency of all grade hypertension (41.6\%) compared to those treated with lenvatinib (65.2\%); the difference was significant ( $\mathrm{OR}=0.31,95 \% \mathrm{CI}=0.23-0.42, P<0.0001$; Figure 2E).

For all grade nausea, the incidence was lower with sorafenib (17.2\%) compared with lenvatinib (34.5\%). Moreover, the difference between the incidence of nausea was significant between sorafenib and lenvatinib $(\mathrm{OR}=0.40$, $95 \% \mathrm{CI}=0.27-0.57, P<0.0001$; Figure $2 \mathrm{~F}$ ).
The frequency of all grade elevated ALT was more common for sorafenib compared with lenvatinib $(12.6 \% \mathrm{vs}$ $0.4 \%, \mathrm{OR}=37.43,95 \% \mathrm{CI}=5.00-277.85, P<0.0001$; Figure $2 \mathrm{G})$. We also identified all grade elevated AST more frequently in patients treated with sorafenib compared with lenvatinib $(11.1 \%$ vs $0.4 \%, \mathrm{OR}=32.54,95 \%$ $\mathrm{CI}=4.34-242.90, P<0.0001$; Figure $2 \mathrm{H})$. For all grade vomiting, sorafenib had a lower incidence compared with lenvatinib $(11.1 \%$ vs $25.9 \%, \mathrm{OR}=0.36,95 \% \mathrm{CI}=0.22-0.58$, $P<0.0001$; Figure 2I).

However, other all grade TRAEs, including diarrhea, weight loss, anorexia, fatigue, and mucositis, showed no significant differences.

\section{Frequency of severe TRAEs (grade $\geq 3$ ) between sorafenib and lenvatinib}

Grade $\geq 3$ hand-foot syndrome was more frequent in patients treated with sorafenib compared with those treated with lenvatinib $(20.6 \%$ vs $3.0 \%$, OR $=8.25,95 \% \mathrm{CI}=4.19-16.24$, $P<0.0001$; Figure $3 \mathrm{~A})$.

However, patients in the sorafenib group experienced a significantly lower frequency of severe weight loss compared with the lenvatinib group $(1.3 \%$ vs $8.1 \%$, OR $=0.15,95 \%$ $\mathrm{CI}=0.04-0.48, P=0.0001$; Figure 3B).

Another severe adverse effect, hypocalcemia, was found at a higher frequency in the sorafenib group (9.2\%) compared with the lenvatinib group (2.2\%). Moreover, the difference between sorafenib and lenvatinib was statistically significant (OR=3.15, 95\% CI=1.30-7.63, $P=0.009$; Figure 3C). For grade $\geq 3$ hypertension, the frequency was lower in the sorafenib group (10.5\%), compared with the lenvatinib group 
A

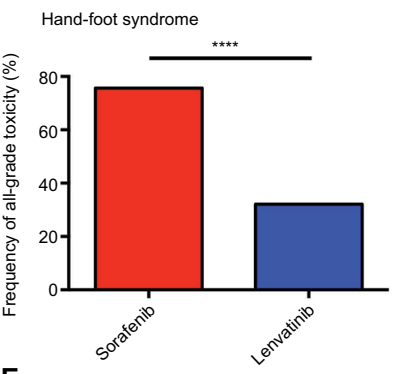

E

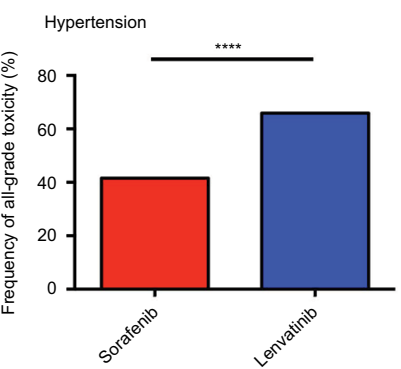

I

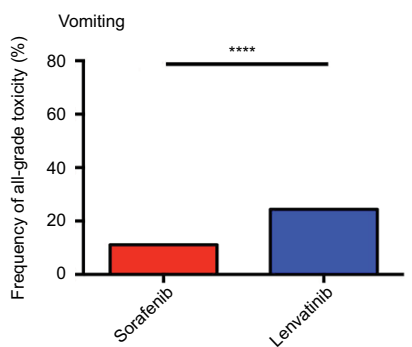

B

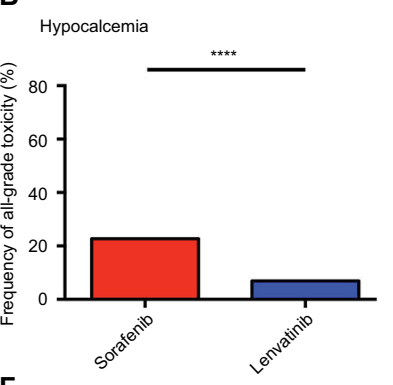

F

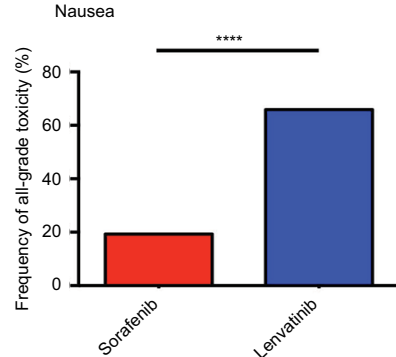

C
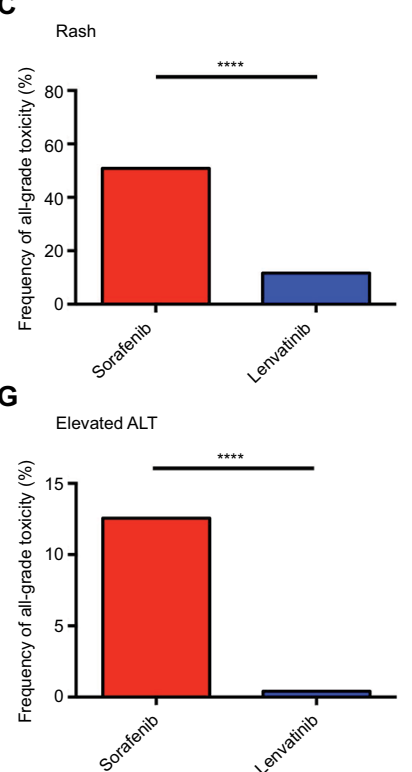

D

Voice change

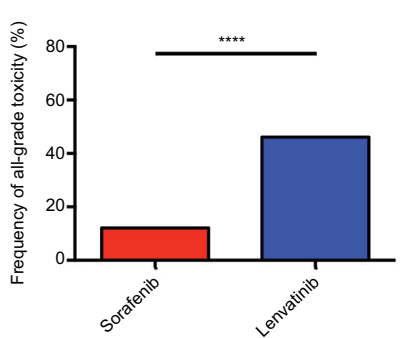

H

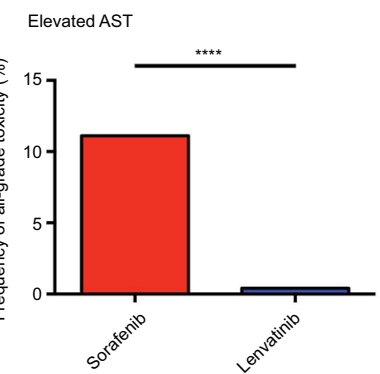

Figure 2 Frequency of all grade toxicity, including hand-foot syndrome (A), hypocalcemia (B), rash (C), voice change (D), hypertension (E), nausea (F), elevated ALT (G), elevated AST $(\mathbf{H})$, and vomiting $(\mathbf{I})$, among sorafenib and lenvatinib.

Note: $* * * * P<0.000$ I indicates statistical significance.

Abbreviations: ALT, alanine aminotransferase; AST, aspartate aminotransferase.

(35.2\%). Statistical significance was observed among the two TKIs (OR $=0.22,95 \% \mathrm{CI}=0.14-0.34, P<0.0001$; Figure 3D).

Likewise, patients who underwent sorafenib treatment experienced a lower frequency of severe nausea compared with those who underwent lenvatinib therapy, with statistical significance ( $0 \%$ vs $1.7 \%$, OR $=0.11,95 \%$ CI $=0.01-2.09$, $P<0.05$; Figure $3 \mathrm{E})$.

For grade $\geq 3$ elevated ALT, a significant difference was observed among the two drugs, with sorafenib exhibiting a higher frequency compared with lenvatinib $(2.9 \%$ vs $0 \%$, $\mathrm{OR}=16.87 .95 \% \mathrm{CI}=0.94-301.50, P=0.007$; Figure $3 \mathrm{~F}$ ).

However, for grade $\geq 3$ diarrhea $(\mathrm{OR}=0.68,95 \% \mathrm{CI}=0.36$ 1.30, $P=0.28$ ), mucositis ( $\mathrm{OR}=0.55,95 \% \mathrm{CI}=0.20-1.51$, $P=0.36)$, fatigue $(\mathrm{OR}=0.61,95 \% \mathrm{CI}=0.31-1.21, P=0.15)$, anorexia $(\mathrm{OR}=0.59,95 \% \mathrm{CI}=0.21-1.61, P=0.36)$, and elevated AST $(\mathrm{OR}=6.37,95 \% \mathrm{CI}=0.30-133.4, P=0.19)$, no significant differences were found when comparing sorafenib and lenvatinib treatment.

\section{Identification of withdrawal toxicity and TRD for sorafenib vs lenvatinib}

The overall frequency of AEs that resulted in withdrawal for sorafenib and lenvatinib was $18.1 \%$ (43/238) and $12.8 \%$ (53/419), respectively. However, the difference between the two drugs was not statistically significant $(\mathrm{OR}=1.52,95 \%$ $\mathrm{CI}=0.98-2.36, P=0.06)$. Furthermore, no significant differences were observed in AEs that resulted in TRD between sorafenib and lenvatinib $(0.8 \%$ vs $1.9 \%, \mathrm{OR}=0.43,95 \%$ $\mathrm{CI}=0.09-2.08, P=0.32)$.

\section{Discussion}

In the past few years, the small molecular TKIs sorafenib and lenvatinib have been investigated for the treatment of advanced or RR-DTC, which was approved by the FDA in 2013 and 2015, respectively. Moreover, several TKIs, including cabozantinib, ${ }^{5}$ vandetinib, ${ }^{7}$ axitinib,${ }^{14}$ and sunitinib ${ }^{15}$ have shown clinical efficiency in the management of 

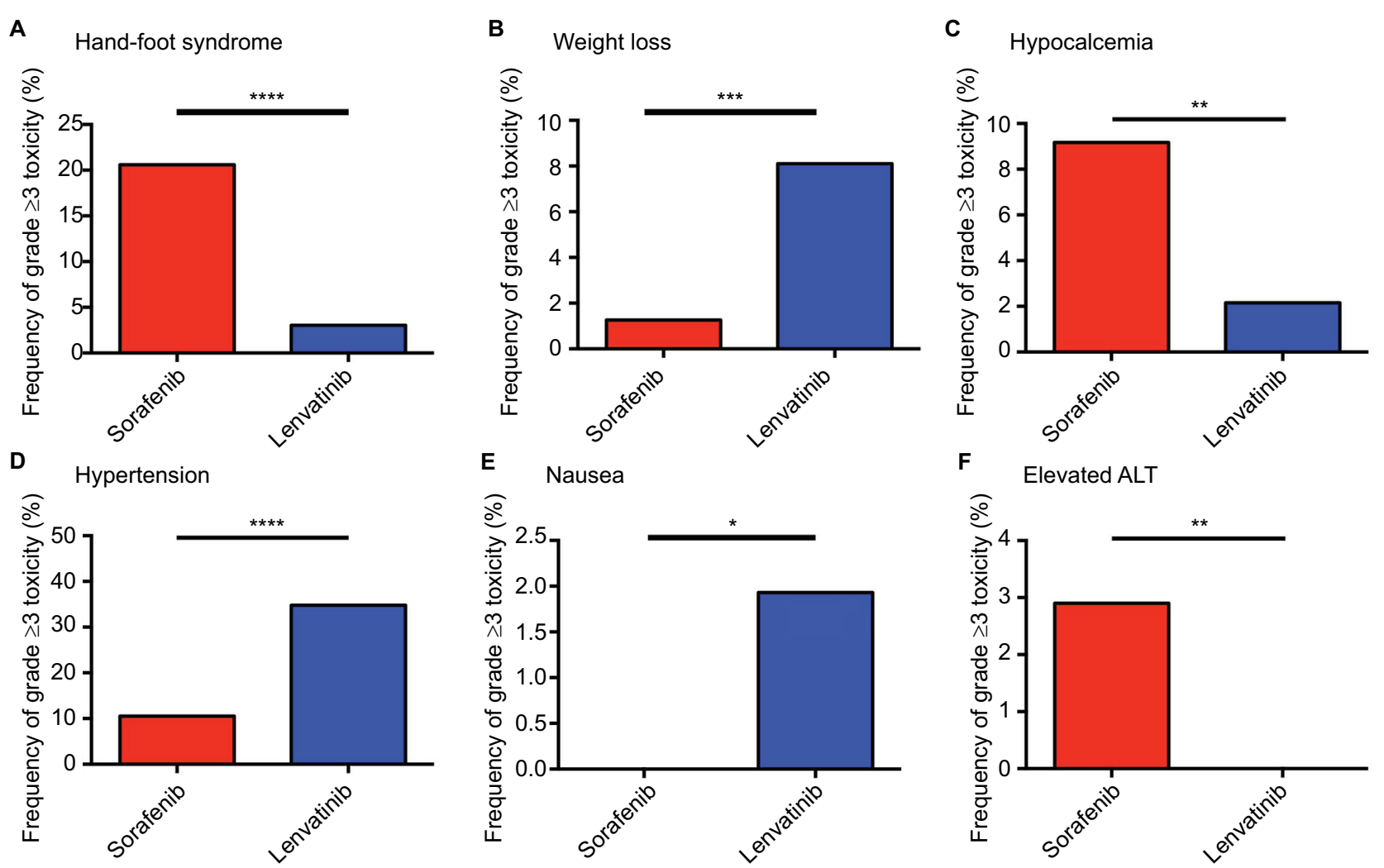

Figure 3 Frequency of grade $\geq 3 \mathrm{AEs}$, including hand-foot syndrome (A), weight loss (B), hypocalcemia (C), hypertension (D), nausea (E), and elevated ALT (F), among sorafenib and lenvatinib.

Note: $* P<0.05, * * P<0.01, * * * P<0.001, * * * * P<0.0001$ indicate statistical significance.

Abbreviations: $A E s$, adverse effects; $A L T$, alanine aminotransferase.

advanced DTC. However, use of these inhibitors has been restricted by the concomitance of adverse effects, such as rash, hypertension, and hand-foot syndrome. The management of alleviating and preventing side effects could promote patients' health-related quality-of-life (HRQoL). Resteghini et a ${ }^{16}$ reported that some TRAEs are preventable, such as skin toxicity and hypocalcemia. However, effective preventative treatments for some TRAEs, including nausea, vomiting, and mucosal toxicities, are not yet available. Therefore, exploring the differences in incidence of TRAEs among different TKI drugs in advanced RR-DTC may assist the early management of the most susceptible patients. Thus, it is significant to determine the frequency of TRAEs to minimize the risk of treatment-related withdrawal or death.

To the best of our knowledge, the current study is the first pooled analysis focused on the differences of TRAEs among sorafenib and lenvatinib. In our study, we found that sorafenib-treated patients experienced a significantly higher frequency of all grade hand-foot syndrome, hypocalcemia, rash, elevated ALT, and elevated AST. Conversely, patients treated with lenvatinib experienced more frequent all grade voice change, hypertension, nausea, and vomiting compared with those treated with sorafenib. For grade $\geq 3$ adverse effects, we found that hand-foot syndrome, hypocalcemia, and elevated ALT were more frequent in sorafenib-treated patients. Moreover, lenvatinib-treated patients had a significantly higher incidence of severe weight loss, hypertension, and nausea.

The side effects of TKIs, such as hypertension, fatigue, and skin problems, are generally manageable. ${ }^{17}$ Furthermore, some researchers have reported that dermatological AEs are not the primary reason for discontinuing treatments. ${ }^{4}$ However, early management is important for improving HRQoL. Liu et a $\mathrm{al}^{18}$ reported that over $55 \%$ of patients need to receive dose reduction and intervention in TKI drug trials. Moreover, in the current study, we identified that the sorafenib group had a higher frequency of withdrawal compared with the lenvatinib group, although the difference in frequency was not statistically significant.

Furthermore, we analyzed the difference in the incidence of life-threatening AEs among the two drugs. However, no significance was noted. Furthermore, TKIs have been associated with long QTc prolongation. ${ }^{19}$ However, no study other than Schneider et al's ${ }^{9}$ has reported the AEs of this cardiac complication. For better use of TKIs, active monitoring of AEs throughout the entire treatment is critical. 
Kiyota et $\mathrm{a}^{20}$ assessed the results of a Japanese population in relation to those in Schlumberger et al's ${ }^{8}$ trial. The treatment efficacy in the Japanese population was similar to the other population. However, a higher incidence of hypertension and a higher dose reduction rate were found in the Japanese population. These results indicate that regional diversity of $\mathrm{AE}$ frequencies and dose modifications may be considered in the use of TKIs.

Our study has several limitations. First, this study assessed the difference in incidence of TRAEs between two FDAapproved drugs, sorafenib and lenvatinib. Other TKI drugs, including cabozantinib, vandetanib, axitinib, and sunitinib, were excluded from the current study. Thus, a large number of patients with a prospective study should be conducted in the future. Second, even though we contacted the corresponding authors, a number of AEs were not analyzed due to a lack of information. Third, the association of specific TRAEs and specific TKI drugs remains unknown. Further studies are needed to investigate the underlying mechanism of this association.

\section{Conclusion}

Our study has shown that different TKI drugs are associated with a highly increased risk of treatment-related toxicity in advanced or RR-DTC. Early interventions and management of TRAEs based on which TKI drugs are applied can minimize the impacts on patients' QoL, better deploying medical resources. Overall, patients and physicians should be familiar with the risks of TRAEs and early management of their side effects to promote patients' QoL.

\section{Acknowledgments}

This research has received a grant from President Foundation of Nanfang Hospital, Southern Medical University (no. 2018C024). The funders played no role in study design, collection, analysis, interpretation of data, writing of the report, or in the decision to submit the paper for publication. They accept no responsibility for the contents.

\section{Author contributions}

STY and STL designed the research. STY and JNG searched and assessed all potential papers. STL and JYL evaluated the data. ZGW and BHS constructed the figure and table. STY and JYL wrote the manuscript. All authors contributed to data analysis, drafting and revising the article, gave final approval of the version to be published, and agree to be accountable for all aspects of the work.

\section{Disclosure}

The authors report no conflicts of interest in this work.

\section{References}

1. Carling T, Udelsman R. Thyroid cancer. Annu Rev Med. 2014;65:125-137.

2. Gruber JJ, Colevas AD. Differentiated thyroid cancer: focus on emerging treatments for radioactive iodine-refractory patients. Oncologist. 2015;20(2):113-126.

3. Kapiteijn E, Schneider TC, Morreau H, Gelderblom H, Nortier JW, Smit JW. New treatment modalities in advanced thyroid cancer. Ann Oncol. 2012;23(1):10-18.

4. Brose MS, Nutting CM, Jarzab B, et al. Sorafenib in radioactive iodine-refractory, locally advanced or metastatic differentiated thyroid cancer: a randomised, double-blind, phase 3 trial. Lancet. 2014;384(9940):319-328

5. Cabanillas ME, de Souza JA, Geyer S, et al. Cabozantinib as salvage therapy for patients with tyrosine kinase Inhibitor-Refractory differentiated thyroid cancer: results of a multicenter phase II international thyroid Oncology Group trial. J Clin Oncol. 2017;35(29):3315-3321.

6. Klein Hesselink EN, Steenvoorden D, Kapiteijn E, et al. Therapy of endocrine disease: response and toxicity of small-molecule tyrosine kinase inhibitors in patients with thyroid carcinoma: a systematic review and meta-analysis. Eur J Endocrinol. 2015;172(5):R215-R225.

7. Leboulleux S, Bastholt L, Krause T, et al. Vandetanib in locally advanced or metastatic differentiated thyroid cancer: a randomised, double-blind, phase 2 trial. Lancet Oncol. 2012;13(9):897-905.

8. Schlumberger M, Tahara M, Wirth LJ, et al. Lenvatinib versus placebo in radioiodine-refractory thyroid cancer. $N$ Engl J Med. 2015;372(7): 621-630.

9. Schneider TC, Abdulrahman RM, Corssmit EP, Morreau H, Smit JW, Kapiteijn E. Long-term analysis of the efficacy and tolerability of sorafenib in advanced radio-iodine refractory differentiated thyroid carcinoma: final results of a phase II trial. Eur J Endocrinol. 2012;167(5):643-650.

10. Balmelli C, Railic N, Siano M, et al. Lenvatinib in advanced radioiodinerefractory thyroid cancer - a retrospective analysis of the swiss lenvatinib named patient program. J Cancer. 2018;9(2):250-255.

11. Nervo A, Gallo M, Samà MT, et al. Lenvatinib in advanced radioiodinerefractory thyroid cancer: a snapshot of real-life clinical practice. Anticancer Res. 2018;38(3):1643-1649.

12. Berdelou A, Borget I, Godbert Y, et al. Lenvatinib for the treatment of radioiodine-refractory thyroid cancer in real-life practice. Thyroid. Epub 2017 Nov 27.

13. Cabanillas ME, Schlumberger M, Jarzab B, et al. A phase 2 trial of lenvatinib (E7080) in advanced, progressive, radioiodine-refractory, differentiated thyroid cancer: a clinical outcomes and biomarker assessment. Cancer. 2015;121(16):2749-2756.

14. Locati LD, Licitra L, Agate L, et al. Treatment of advanced thyroid cancer with axitinib: phase 2 study with pharmacokinetic/pharmacodynamic and quality-of-life assessments. Cancer. 2014;120(17):2694-2703.

15. Marotta V, di Somma C, Rubino M, et al. Second-line sunitinib as a feasible approach for iodine-refractory differentiated thyroid cancer after the failure of first-line sorafenib. Endocrine. 2015;49(3): 854-858.

16. Resteghini C, Cavalieri S, Galbiati D, et al. Management of tyrosine kinase inhibitors (TKI) side effects in differentiated and medullary thyroid cancer patients. Best Pract Res Clin Endocrinol Metab. 2017;31(3): 349-361.

17. Sherman SI. Targeted therapy of thyroid cancer. Biochem Pharmacol. 2010;80(5):592-601.

18. Liu JW, Chen C, Loh EW, et al. Tyrosine kinase inhibitors for advanced or metastatic thyroid cancer: a meta-analysis of randomized controlled trials. Curr Med Res Opin. 2018;34(5):795-803.

19. Ghatalia P, Je Y, Kaymakcalan MD, Sonpavde G, Choueiri TK. QTc interval prolongation with vascular endothelial growth factor receptor tyrosine kinase inhibitors. Br J Cancer. 2015;112(2):296-305.

20. Kiyota N, Schlumberger M, Muro K, et al. Subgroup analysis of Japanese patients in a phase 3 study of lenvatinib in radioiodine-refractory differentiated thyroid cancer. Cancer Sci. 2015;106(12):1714-1721. 


\section{Supplementary materials}

Our search strategy on the Pubmed was as follows:

(()((sorafenib[Title/Abstract]) OR lenvatinib [Title/ Abstract]) AND differentiated thyroid cancer) AND English[Language])) AND (“2008/01/01”[Date - Publication] : “2018/05/30”[Date - Publication]).

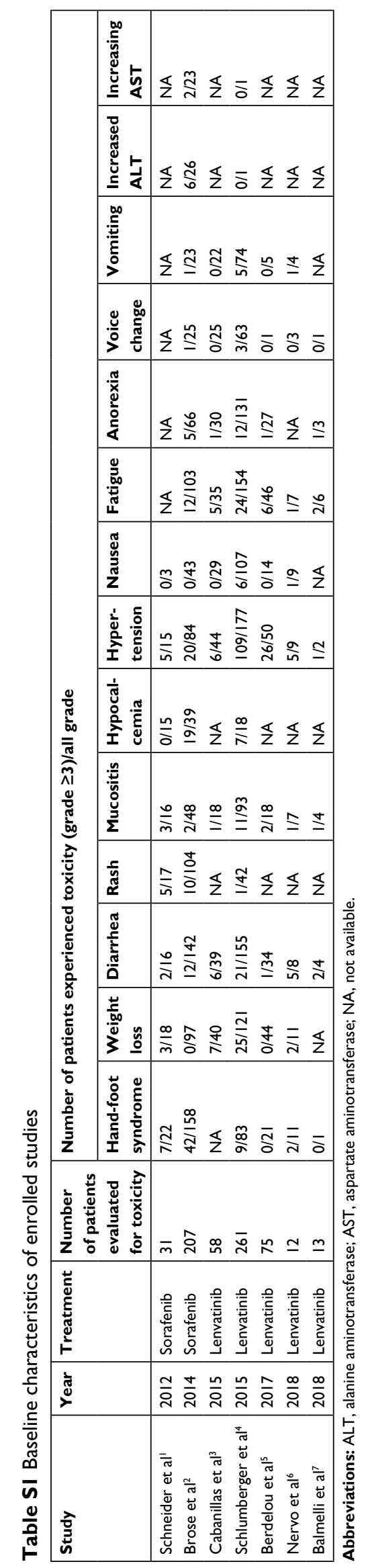


Table S2 Risk of bias in enrolled studies

\begin{tabular}{|c|c|c|c|c|c|c|}
\hline Study & $\begin{array}{l}\text { Adequate sequence } \\
\text { generation }\end{array}$ & $\begin{array}{l}\text { Allocation } \\
\text { concealment }\end{array}$ & Blinding & $\begin{array}{l}\text { Incomplete outcome } \\
\text { data addressed }\end{array}$ & $\begin{array}{l}\text { Free selective } \\
\text { reporting }\end{array}$ & $\begin{array}{l}\text { Free of } \\
\text { other bias }\end{array}$ \\
\hline Schneider et al' & Yes & No & Yes & No & No & No \\
\hline Brose et $\mathrm{al}^{2}$ & Yes & Yes & Yes & No & No & No \\
\hline Cabanillas et $\mathrm{al}^{3}$ & Yes & No & Yes & No & No & No \\
\hline Schlumberger et $\mathrm{al}^{4}$ & Yes & Yes & Yes & No & No & No \\
\hline Berdelou et $\mathrm{al}^{5}$ & Yes & No & No & No & No & No \\
\hline Nervo et $\mathrm{al}^{6}$ & Yes & No & No & No & No & No \\
\hline Balmelli et $\mathrm{al}^{7}$ & Yes & No & No & No & No & No \\
\hline
\end{tabular}

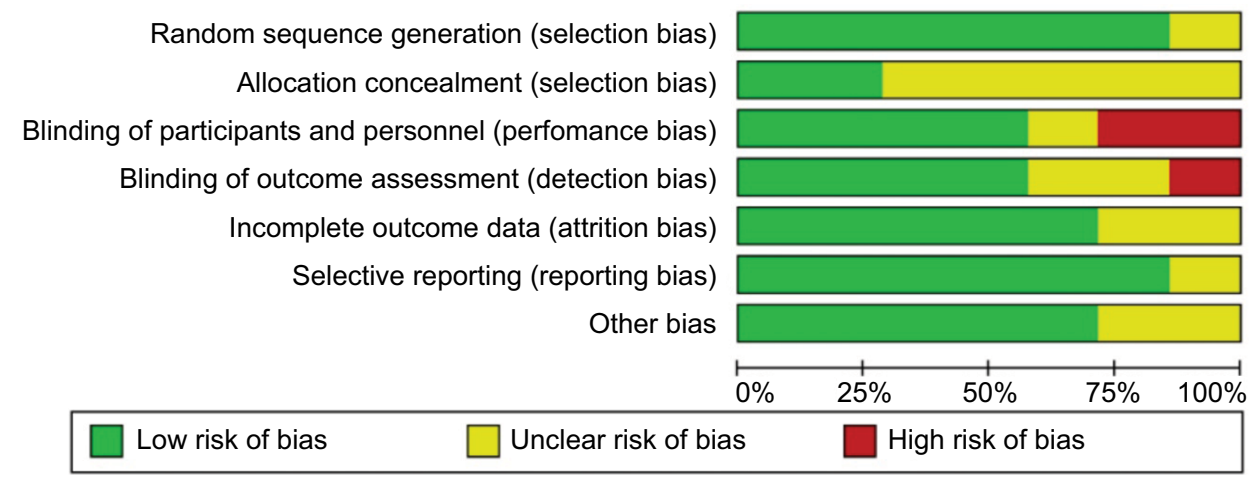

Figure SI Risk of bias graph

\section{References}

1. Schneider TC, Abdulrahman RM, Corssmit EP, Morreau H, Smit JW, Kapiteijn E. Long-term analysis of the efficacy and tolerability of sorafenib in advanced radio-iodine refractory differentiated thyroid carcinoma: final results of a phase II trial. Eur J Endocrinol. 2012;167(5): 643-650.

2. Brose MS, Nutting CM, Jarzab B, et al. Sorafenib in radioactive iodinerefractory, locally advanced or metastatic differentiated thyroid cancer: a randomised, double-blind, phase 3 trial. Lancet. 2014;384(9940):319-328.

3. Cabanillas ME, Schlumberger M, Jarzab B, et al. A phase 2 trial of lenvatinib (E7080) in advanced, progressive, radioiodine-refractory, differentiated thyroid cancer: a clinical outcomes and biomarker assessment. Cancer. 2015;121(16):2749-2756.
4. Schlumberger M, Tahara M, Wirth LJ, et al. Lenvatinib versus placebo in radioiodine-refractory thyroid cancer. $N$ Engl J Med. 2015;372(7): 621-630.

5. Berdelou A, Borget I, Godbert Y, et al. Lenvatinib for the treatment of radioiodine-refractory thyroid cancer in real-life practice. Thyroid. Epub 2017 Nov 27.

6. Nervo A, Gallo M, Samà MT, et al. Lenvatinib in advanced radioiodinerefractory thyroid cancer: a snapshot of real-life clinical practice. Anticancer Res. 2018;38(3):1643-1649.

7. Balmelli C, Railic N, Siano M, et al. Lenvatinib in advanced radioiodinerefractory thyroid cancer - a retrospective analysis of the swiss lenvatinib named patient program. $J$ Cancer. 2018;9(2):250-255.
Cancer Management and Research

\section{Publish your work in this journal}

Cancer Management and Research is an international, peer-reviewed open access journal focusing on cancer research and the optimal use of preventative and integrated treatment interventions to achieve improved outcomes, enhanced survival and quality of life for the cancer patient. The manuscript management system is completely online and includes

\section{Dovepress}

a very quick and fair peer-review system, which is all easy to use. Visit http://www.dovepress.com/testimonials.php to read real quotes from published authors. 